\title{
Performance evaluation of UE-controlled intelligent handover algorithm for natural disaster
}

\author{
Azita Laily Yusof, Ainnur Eiza Azhar, Norsuzila Ya'acob \\ Wireless Communication Technology Group, Faculty of Electrical Engineering, \\ Universiti Teknologi MARA, Malaysia
}

\begin{abstract}
Article Info
ABSTRACT

Article history:

Received Jan 29, 2020

Revised Apr 1, 2020

Accepted Apr 15, 2020

This paper proposes a UE-controlled intelligent handover algorithm for natural disaster. In this handover algorithm, two variables known as modified received signal strength (RSSm) and left over power (LoP) are identified. The RSSm is an improved formulation from RSS where distance fraction coefficient has been introduced. The fraction coefficient of 0.2 is used where the affected areas is reduced so that the users can receive good signal quality due to its location near to the base station. Meanwhile, the LoP also has been investigated to control power consumption of base station. In this research,

Keywords: $80 \%$ RSSm and 20\% LoP has been chosen for the proposed handover

Fraction coefficient

Handover

Left over power

Natural disaster algorithm as it can maintain good quality of service (QoS) for all users and also can prolong battery life. From the simulation results obtained, the average number of handovers for the proposed handover algorithm outperformed the conventional natural disaster handover algorithm.
\end{abstract}

Power consumption

Copyright $₫ 2020$ Institute of Advanced Engineering and Science. All rights reserved.

Corresponding Author:

Azita Laily Yusof,

Wireless Communication Technology Group,

Faculty of Electrical Engineering,

Universiti Teknologi MARA, Shah Alam, Malaysia.

Email: laily012001@yahoo.com

\section{INTRODUCTION}

Natural disaster is an unpredicted event that will disturb the operation of mobile communications. One of critical damage is the dis-function of base stations due to hardware failure [1] and power outage. Although, each base station is equipped with battery backup, it can only last several hours depend on its power consumption [2]. During natural disaster, the affected areas will heavily congested with more users [3] as they need to connect to the base station due to search and rescue operation [4]. When more users connected to the affected base station, more power is needed to operate [5] and automatically battery power will decreases significantly.

The mobile operators need to propose a countermeasure to make sure users in the affected area receive satisfying network quality while prolong battery life of base station [6-7]. If there is no countermeasure taken, the battery life becomes critical, eventually the traffic will becomes congestion, user experience may degrades and rescue operation will becomes difficult. As example, a smartphone-based post-disaster management mechanism in natural-disaster affected areas using WiFi tethering can be use for search and rescue operation. The technique make the smartphones into temporary mobile hotspots and provide internet connectivity to other WiFi-enabled clients during disaster [8]. In addition, in [9], the author adopted device-to-device (D2D) communication to extend cellular coverage during natural disaster by enabling nearby UEs to directly communicate with each other by passing the cellular base stations. This technique can make the UE to serve as a decode-and-forward relay node to communicate with other UEs. It shows the need to have a good quality of mobile communication network although the affected areas has power outage [10]. 


\section{RESEARCH WORKS}

Several researchers had proposed various handover algorithms to maintain users' experience. According to [11], handover is defined as a mechanism that transfers an ongoing call from one cell to another as a user moves through the coverage area of a cellular system. If handover does not occur quickly, the QoS may degenerate below an acceptable level [12]. Authors in [13, 14] investigated handover algorithms in LTE high-speed railway networks. The papers focused on optimization of A3 event-based HO algorithms especially for high-speed velocity which the link quality may worsen and the wireless channel environment may become unstable with the increase of velocity. The advantage of the technique is it considers several variables which are reference signal received power (RSRP), reference signal received quality (RSRQ) and the rate of cell resources.

Researchers in $[15,16]$ proposed handover algorithm with received signal strength that can reduce the number of average handovers per UE per second, shorter total system delay whilst maintaining a higher total system throughput. However, both techniques limit the number of handovers although its improve quality of wireless communication; but for natural disaster scenario, more users are need to connect to base station for the rescue operation purpose. On the other hand, there are several articles focus on load balancing in 3GPP LTE network. Papers in [17, 18] investigated auto-tuning of LTE mobility and self-optimizing networks (SON) algorithms, respectively. In [19], the author proposed Heaviest-First Load Balancing algorithm. The results shown that the technique balance the load in the entire network, however, during natural disaster, unbalance load may occur due to traffic congestion. Meanwhile, author in [20] introduced an energy-centric handover algorithm as handover decision to minimize the power consumption of LTE UE and cell in LTE-femtocell network. However, this technique increased the LTE network signaling.

Author in [21] proposed Limited CoMP Handover Algorithm with joint processing (JP) in CoMP technique to overcome capacity problem for high congested network. Although the system throughput improved, the system delay also increased due to all the packets need to be buffered longer in the queue to get transmitted to all the UEs. Other than that, the author in [22] proposed a radio problem detection based rescue handover for $3 \mathrm{G}$ long term evolution (LTE) by adopting radio link failure (RLF) detection mechanism. This technique can decrease the amount of RLFs, hence improving the handover and system performance. However, this technique applies the non-regular simulation scenario instead of the regular macro defined by the 3GPP. Another article in [23] introduced two variables which are UE's distance of motion (DoM) relative to a NeNB and left over power (LoP) of NeNBs as handover decision to maintain the user's QoS during disaster and provided power control for battery backup. However, the technique is complicated as it used angle of divergence (AOD) for determining DoM value.

This paper investigates the continuity of the paper [23] and propose a handover algorithm that use receive signal strength and left over power. Both parameters will be use to compute weighted-average score (WAS) that is use as handover decision to improve signal quality and prolong the battery life by reduces the power consumption. The rest of the paper is organized into six different sections. Section 2 discusses previous related works. Section 3 presents the proposed handover algorithm and Section 4 describes on simulation description. Simulation results and discussion are explained in Section 5 and finally conclusion is drawn in Section 6.

\section{PROPOSED HANDOVER ALGORITHM}

This section explains detail on the proposed handover algorithm. When an UE is moving away from the serving cell, there are two conditions that will occur which are natural disaster and no natural disaster conditions. The no natural disaster represents scenario which used received signal strength only to handover. On the other hand, the natural disaster condition takes left over power of a base station into account as during the incident, the base station uses battery backup as the area power outage may occur. Hence, the affected base stations need to control its battery power so that users can have satisfied user's experience although in natural disaster condition.

For natural disaster, the WAS value which is the summation of number of users that connected to a base station, the distance value of an UE to the serving base stations and the weight of RSS and LoP is calculated to satisfy WAS $>\mathrm{WAS}_{\mathrm{TH}}$. When the WAS is greater than the $\mathrm{WAS}_{\mathrm{TH}}$, the UE is allowed to progress toward the next step. The value of WAS $\mathrm{TH}_{\mathrm{TH}}$ is chosen from the value of WAS with Wrss $=0.8$ and wlop=0.2 for 100 users because it maintain the QoS of UE and only provides $20 \%$ of battery backup, hence more users can connected to the affected base station. In this work, the WAS is based on distance of UE from the serving base station and left over power of base station during natural disasters. The modified WAS for each NeNB can be expressed as:

$$
W A S(\text { modified })=S_{\text {LoP }}\left(N e N B_{i}\right) \times W_{\text {Lop }}+S_{R S S_{\_} \text {modified }}\left(N e N B_{i}\right) \times W_{R S S}
$$


where $S_{L O P}$ is the left over power score of a battery, $S_{R S S \text { modified }}$ is RSSmodified score of UE with value of $0.8(1<\mathrm{d}<50), W_{L O P}$ and $W_{R S S}$ are the weight of LOP and RSS, respectively with value of $80 \%$ wRSS and $20 \%$ wLOP that means location of UE is near to BS with only small percentage of battery backup in natural disaster incidents. The $S_{L O P}$ can be defined as [23] with $\mathrm{N}$ is total of subscribers and $\mathrm{n}$ is number of connection to the base station:

$$
S_{\text {Lop }}=\left[\left(N-\frac{n}{N}\right)\right] \times \text { Ratedpower of the battery }
$$

In continuity, the handover algorithm decided to check the RSS of the UEs before initiating the handover call to another BS. It must satisfied condition for $\mathrm{RSSm}<\mathrm{RSS}_{\mathrm{TH}}$. If the condition of the handover threshold serving value was not satisfied, the process will return to calculate the new distance of UE and base stations and then calculate the RSS again. Channels will be released once the call is finished and the simulation will be terminated at the end of the simulation times. The RSSm is the modified expression of RSS that can improve the signal strength of an UE. The modified received signal strength $\left(R S S_{\text {modified }}\right)$ in decibel is given by:

$$
R S S_{\text {modified }}=P_{t}+G_{t}+G_{r}-P L_{\text {modified }}
$$

where $P_{t}$ is the transmitted power, $G_{t}$ is the transmitted antenna gain, $G_{r}$ is the received antenna gain and $P L_{\text {modified }}$ is the modified path loss between the transmitter and the UE. The modified path loss for this work is adopted two-ray path loss and can be defined as:

$$
P L_{\text {modified }}=20 \times \log \left(\alpha \times \frac{d^{2}}{h_{t}} \times h_{r}\right)
$$

where $d$ is the distance between the eNodeB and UE. The $h_{t}$ and $h_{r}$ are height of transmitter and UE, respectively. The $\alpha$ is the proposed coefficient namely as distance fraction coefficient. The $\alpha$ of 0.2 is used as the optimal value as it reduces the user's area serving hence giving it much lower path loss value to the system. Hence, it gave the highest RSS value of UE compare to others. Then, the handover will check the availability of channels and bandwidth at the target base station. If $\operatorname{Load}<\operatorname{Load}_{\mathrm{TH}}$, the UE will be connected automatically to the target base station. If the above conditions are not satisfied, the UE will return to the step of checking WAS and RSS measurement again. Under this condition, if the channel at the target traffic load is at maximum, the UE is prepared to initiate the handover to another base station.

For no natural disaster, the handover algorithm decided to check the RSS of the UEs only. If the RSSm<RSSTH, the UE is acceptable for next condition. If the above condition is not satisfied, the UE will then check the traffic load at base station. If the traffic loads condition at the target base station has available channels or bandwidths, the UE will automatically handover to macrocell. If the traffic loads condition at the target base station does not have available channels or bandwidths, the UE then return to the step of checking speed and RSS measurement again. The channels will be released once the call finished

\begin{tabular}{|c|c|}
\hline Steps & Description \\
\hline Step 1 & RSS measurement of UE. \\
\hline Step 2 & $\begin{array}{l}\text { Natural disaster incident? If yes, trigger the new handover algorithm for natural disaster. Otherwise, the no } \\
\text { natural handover algorithm is used. }\end{array}$ \\
\hline Step 3 & $\begin{array}{l}\text { UE position near target base station? If yes, go to step } 4 \text { for natural disaster. For no natural disaster, go to } \\
\text { step } 5 \text {. Otherwise go to step } 1 \text {. }\end{array}$ \\
\hline Step 4 & $\begin{array}{l}\text { Calculate the value of the criterion function for natural disaster WAS }>\text { WAS } S_{\mathrm{TH}} \text {. When the triggering } \\
\text { condition as shown above is fulfilled for the entire TTT time duration, go to step 5. Otherwise go to step } 1 \text {. }\end{array}$ \\
\hline Step 5 & $\begin{array}{l}\text { Calculate the value of the criterion function } \mathrm{RSSm}<\mathrm{RSS}_{\mathrm{TH}} \text {. When the triggering condition as shown above } \\
\text { is fulfilled for the entire TTT time duration, go to step } 6 \text {. Otherwise go to step } 1 \text {. }\end{array}$ \\
\hline Step 6 & Available bandwidth? If yes, go to step 7. Otherwise go to step 1 . \\
\hline Step 7 & $\begin{array}{l}\text { Calculate the value of the criterion function for natural disaster Load }<\operatorname{Load}_{\mathrm{TH}} \text {. When the triggering condition } \\
\text { as shown above is fulfilled for the entire TTT time duration, handover to target base station. }\end{array}$ \\
\hline
\end{tabular}
and terminated at the end of the simulation times. Table 1 shows the procedure of the proposed handover algorithm.

Table 1. Procedure of the proposed handover algorithm

The system performance of the proposed handover algorithm in natural disaster and no natural disaster are evaluated on the basis of average handover per number of UE based on UE speed [15] and UE 
movement [24]. According to [25], the transmitter's location is an important factor for the users receive satisfying signal quality. The average handover represents the number of handover occurs during a simulation is expressed as [15]:

$$
H O_{\text {avg }}=\frac{H O_{T o t a l}}{J \times T}
$$

where $\mathrm{HO}_{\text {avg }}$ is the average handover per UE per second, $\mathrm{HO}_{\text {Total }}$ is the total number of successful handover from source to target cell while maintaining the on-going data transmission, $J$ is the total number of users and $T$ is total simulation time.

\section{SIMULATION DESCRIPTION}

For this work, the area was simulated as a homogeneous network with the UE is moving in certain movement throughout the network. As shown in Figure 1, the simulation model was developed using 29 hexagon cells with 29 of macro base station (MBS) and represented by black triangle. The framework was modelled as illustrated in order to see the movement of UE within the network. The radius of MBS deployed was estimated to be in range of $1 \mathrm{~km}$ following as the 3GPP specifications in [26] for urban macro network and 20 to 100UEs were generated in this simulation model. In this work, handover decision is based on RSSm and WAS for an UE. The RSSm is the modified expression of RSS that can improve the signal strength of an UE. Meanwhile, the WAS is based on distance of UE from the serving base station and left over power of base station during natural disasters. System parameters used in the simulation are given in Table 2.

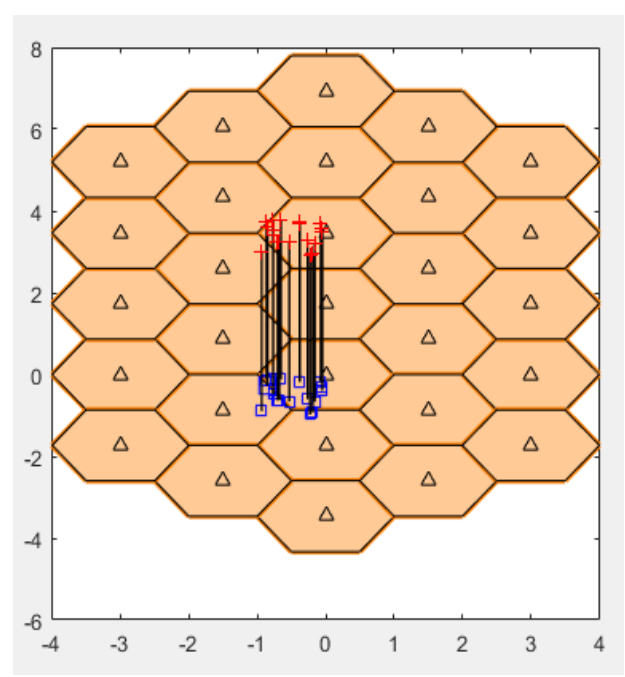

Figure 1. Proposed simulation of handover algorithm
Table 2. Simulation parameters and its value

\begin{tabular}{ll}
\hline \multicolumn{1}{c}{ Parameter } & \multicolumn{1}{c}{ Value } \\
\hline Transmitted power of a BS, $P_{t}$ & $46 \mathrm{dBm}[27]$ \\
Transmitter antenna gain, $G_{t}$ & $15(\mathrm{dbi})$ for downlink \\
Receiver antenna gain, $G_{r}$ & 0 (dbi) for downlink \\
Distance fraction coefficient, $\alpha$ & 0.2 \\
Distance from UE to BS, $d$ & minimum distance $35 \mathrm{~m}$ \\
& {$[26]$} \\
Transmitter's antenna height, $h_{t}$ & $30 \mathrm{~m}[26]$ \\
Receiver's antenna heights, $h_{r}$ & $1.5 \mathrm{~m}[26]$ \\
UE speed [km/h] & $3,30,120$ [15] \\
UE movement & North, South, West, East, \\
& South-East, South-West, \\
& North-East and North-West \\
Number of connection, $\mathrm{n}$ & $20,40,60,80$ and 100 \\
Maximum of N connections at any time & 100 \\
without any degradation in the QoS, N & \\
Modified RSS score for neighboring eNB,, & 0.8 \\
$S_{R S S}$ modified $\left(N e N B_{i}\right)$ & \\
Weight of lop, $W_{L o P}$ & 0.2 \\
Weight of RSS, $W_{R S S}$ & 0.8 \\
Threshold Modified RSS, RSSm & $-68 \mathrm{~dB}$ \\
Threshold WAS, WAS & 0.6 \\
Load MBS Threshold, Load & $90 \%[28]$ \\
\hline
\end{tabular}

\section{SIMULATION RESULTS}

This section explains the simulation results and its analysis for the proposed handover algorithm between natural disaster and no natural disaster. For no natural disaster, the RSS prediction method will give full weight (i.e., 100\%) while LoP not considered at all (i.e., 0\% weight). Meanwhile, for natural disaster, the RSS prediction method will give $80 \%$ full weight and LoP is $20 \%$ weight.

Figure 2 shows an average number of handovers versus number of UEs for proposed handover algorithm in natural disaster and no natural disaster. The number of UE is increases from 20 to 100 with stepsize 20. The result demonstrated that the average number of handovers grow larger with the number of UEs for both algorithms. It is because of more handovers take place when there are more users. However, the proposed handover algorithm for natural disaster has higher average number of handovers as compare to no natural disaster because of improvement in received signal strength value. On the other hand, Figure 3 shows an average number of handovers versus UE speed for the proposed handover algorithm in natural disaster and no natural disaster. The number of UE is fixed to 100 UEs with different UE speed which are $3 \mathrm{~km} / \mathrm{h}, 30 \mathrm{~km} / \mathrm{h}$ and $120 \mathrm{~km} / \mathrm{h}$. The result demonstrated that the average number of handovers grow larger with the UE speed for 
both algorithms. It is because of UE at high speed has faster initiation time take place than low speed. In addition, the proposed algorithm has reduces the ping-pong handovers at high speed due to the improvement of received signal strength value.

Figure 4 shows an average number of handovers versus UE movement for the proposed handover algorithm in natural disaster and no natural disaster. The number of UE is fixed to 100 UEs with different UE movement which are East, West, North, South, South-East, South-West, North-West and North-East. The result demonstrated that the average number of handovers in natural disaster have similar value. It is due to improvement of received signal strength. By comparing average number of handovers with no natural disaster, it has lower value due to poor received signal strength.

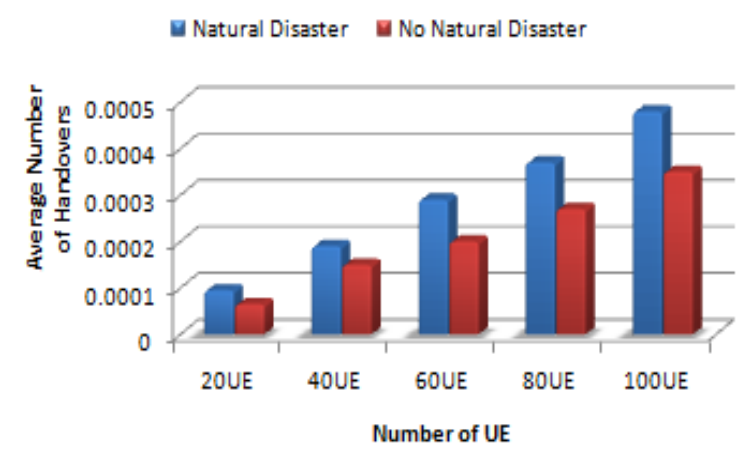

Figure 2. Average number of handovers according to number of UE

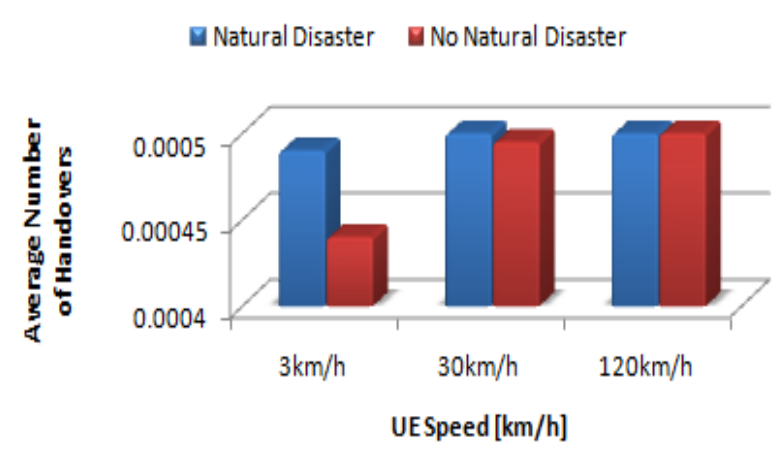

Figure 3.Average number of handovers according to UE speed

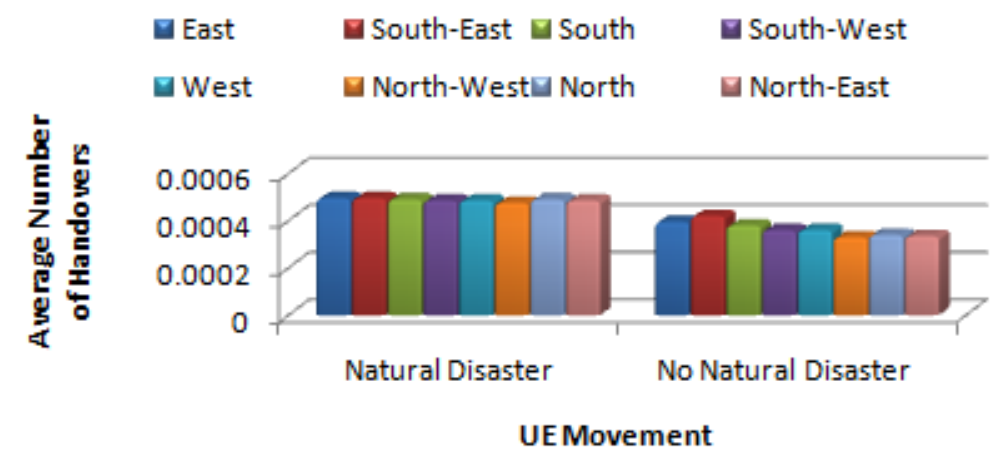

Figure 4. Average number of handovers according to UE movement

\section{CONCLUSION}

During natural disaster, mobile communications may facing problems such as traffic congestion, power outage and critical hardware failure. This paper proposed a UE-controlled intelligent handover algorithm for natural disaster which incorporated two variables known as RSS and LoP. The 80\% RSS and $20 \%$ LoP have been chosen for this algorithm as it can provides good quality of signal, together with $20 \%$ of battery power usage. From the simulation results of average number of handovers versus number of UE, the proposed handover algorithm outperformed the no natural disaster handover algorithm.

\section{ACKNOWLEDGEMENTS}

This paper is part of research work that supported by the FRGS grant no. FRGS/1/2014/TK03/UITM/02/5 and Faculty of Electrical Engineering, UiTM Shah Alam. 


\section{REFERENCES}

[1] J. Huang, Y. Lien, and C. Wang, "Design of Multi-Path Network Topology for Contingency Cellular Network," in 2015 2nd International Conference on Information and Communication Technologies for Disaster Management (ICT-DM), Rennes, pp. 103-108, 2015.

[2] Y. Lien and K. Huang, "Cross Network Topology Design for Contingency Cellular Network," in 2014 IEEE Canada International Humanitarian Technology Conference - (IHTC), Montreal, QC, pp. 1-5, 2014.

[3] R. Austin, P. Bull, and S. Buffery, "A Raspberry Pi Based Scalable Software Defined Network Infrastructure for Disaster Relief Communication," in 2017 IEEE 5th International Conference on Future Internet of Things and Cloud (FiCloud), Prague, pp. 265-271, 2017.

[4] J. Huang, Y. Wu, and Y. Lien, "Bandwidth Allocation of Contingency Cellular Network," in 2013 16th International Symposium on Wireless Personal Multimedia Communications (WPMC), Atlantic City, NJ, pp. 1-6, 2013.

[5] H. Zhang, C. Jiang, R. Q. Hu, and Y. Qian, "Self-Organization in Disaster-Resilient Heterogeneous Small Cell Networks," IEEE Network, vol. 30, no. 2, pp. 116-121, 2016.

[6] K. Ali, H. X. Nguyen, V. Quoc-Tuan, S. Purav, and C. Zheng, "Disaster Management Using D2D Communication With Power Transfer and Clustering Techniques," IEEE Access, vol. 6, pp. 14643-14654, 2018.

[7] T. Nguyen-duc and E. Kamioka, "An SDN Approach for an Energy-Efficient Heterogeneous Communication Network in Disaster Scenarios," International Journal of Wireless \& Mobile Networks, vol. 8, no. 6, pp. 1-18, 2016.

[8] S. K. Ray, Roopak Sinha, and S. K. Ray, "A Smartphone-based Post-Disaster Management Mechanism Using WiFi Tethering," in 2015 IEEE 10th Conference on Industrial Electronics and Applications (IClEA), Auckland, pp. 966-971, 2015.

[9] H. Gao, Y. Shen, and B. Yang, "D2D Communication for Disaster Recovery in Cellular Networks," in 2017 International Conference on Networking and Network Applications (NaNA), Kathmandu, pp. 292-295, 2017.

[10] A. Syahira, M. Anuar, W. N. W. Muhamad, D. M. Ali, S. S. Sarnin, and N. A. Wahab, "A review on link adaptation techniques for energy efficiency and QoS in IEEE802.11 WLAN," Indonesian Journal of Electrical Engineering and Computer Science, vol. 17, no. 1, pp. 331-339, 2020.

[11] G. P. Pollhi, "Trends in Handover Design," IEEE Communications Magazine, vol. 34, no. 3, pp. 82-90, 1996.

[12] T. A. Assegie and H. D. Bizuneh, "Improving network performance with an integrated priority queue and weighted fair queue scheduling," Indonesian Journal of Electrical Engineering and Computer Science, vol. 19, no. 1, pp. 241-247, 2020.

[13] L. Luan, M. Wu, J. Shen, J. Ye, and X. He, "Optimization of handover algorithms in LTE high-speed railway networks,” Int. J. Digit. Content Technol. its Appl., vol. 6, no. 5, pp. 79-87, 2012.

[14] F. Yang, H. Deng, F. Jiang, and X. Deng, "Handover Optimization Algorithm in LTE High-Speed Railway Environment," Wireless Personal Communications, vol. 84, no. 2, pp. 1577-1589, 2015.

[15] C.-C. Lin, K. Sandrasegaran, H. A. M. Ramli, and R. Basukala, "Optimized Performance Evaluation of LTE Hard Handover Algorithm with Average RSRP Constraint," International Journal of Wireless \& Mobile Networks $(I J W M N)$, vol. 3, no. 2, pp. 1-16, 2011.

[16] M. Anas, F. D. Calabrese, P. E. Mogensen, C. Rosa, and K. I. Pedersen, "Performance evaluation of received signal strength based hard handover for UTRAN LTE," 2007 IEEE 65th Vehicular Technology Conference-VTC2007Spring, Dublin, pp. 1046-1050, 2007.

[17] R. Nasri and Z. Altman, "Handover Adaptation for Dynamic Load Balancing in 3GPP Long Term Evolution Systems," 5th International Conference Advanced Mobile Computing and Multimedia, pp. 145-154, 2007.

[18] A. Lobinger, S. Stefanski, T. Jansen, and I. Balan, "Coordinating handover parameter optimization and load balancing in LTE self-optimizing networks," 2011 IEEE 73rd Vehicular Technology Conference (VTC Spring), Yokohama, pp. 1-5, 2011.

[19] H. Wang, L. Ding, P. Wu, Z. Pan, N. Liu, and X. You, "Dynamic Load Balancing and Throughput Optimization in 3GPP LTE Networks," in IWCMC '10: Proceedings of the 6th International Wireless Communications and Mobile Computing Conference, pp. 939-943, 2010.

[20] D. Xenakis, N. Passas, and C. Verikoukis, "An energy-centric handover decision algorithm for the integrated LTE macrocell-femtocell network," Computer Communications, vol. 35, no. 14, pp. 1684-1694, 2012.

[21] C. C. Lin, K. Sandrasegaran, X. Zhu, and Z. Xu, "Limited CoMP Handover Algorithm for LTE-Advanced," Journal of Engineering (United Kingdom), vol. 2013, pp. 1-9, 2013.

[22] J. Puttonen, J. Kurjenniemi, and O. Alanen, "Radio problem detection assisted rescue handover for LTE," 21st Annual IEEE International Symposium on Personal, Indoor and Mobile Radio Communications, Instanbul, pp. 1752-1757, 2010.

[23] S. K. Ray, W. Liu, H. Sirisena, S. K. Ray, and D. Deka, "An Energy Aware Mobile-Controlled Handover Method for Natural Disaster Situations," in 2013 Australasian Telecommunication Networks and Applications Conference (ATNAC), Chirestchurch, pp. 130-135, 2013.

[24] N. Arbain and Z. Kasiran, "Conceptual mobility model of vertical handover decision in heterogeneous networks," Indonesian Journal of Electrical Engineering and Computer Science, vol. 13, no. 3, pp. 1143-1151, 2019.

[25] J. M. Ruiz Avilés, M. Toril, and S. Luna-Ramírez, "A Femtocell Location Strategy for Improving Adaptive Traffic Sharing in Heterogeneous LTE Networks," EURASIP Journal on Wireless Communications and Networking, vol. 2015, no. 38, pp. 1-13, 2015.

[26] 3GPP, "Technical Report 25.996," 2011.

[27] A. L. Yusof, Z. Azren, M. Kamarul, A. E. Azhar, N. Ya, and A. Idris, "Analyzing of Power Control Technique in LTE-Advanced Femtocell Network," Journal of Scientific Research and Development, vol. 3, no. 2, pp. 98-105, 2016.

[28] F. Zhou, L. Feng, P. Yu, and W. Li, "A Load Balancing Method in Downlink LTE Network based on Load Vector Minimization," in 2015 IFIP/IEEE International Symposium on Integrated Network Management (IM), Ottawa, ON, pp. 525-530, 2015. 


\section{BIOGRAPHIES OF AUTHORS}

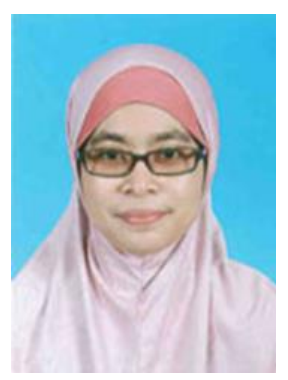

Azita Laily Yusof is an Associate Professor in the Department of Communication Engineering, Universiti Teknologi MARA (UiTM). In 2011, she was awarded a Ph.D degree in Electrical, Electronic \& Systems Engineering from University Kebangsaan Malaysia (UKM). She also obtained her M. Eng. degree from University Kebangsaan Malaysia in Communications \& Computer Engineering in 2000. Previously, she obtained her first degree from University Kebangsaan Malaysia with Honours in Electrical, Electronic \& Systems Engineering in 1999. She is a group member of Wireless Communication Technology Group (WiCoT) at UiTM and a member of IEEE Communications Society. She has been awarded 17 research grants funded by the government and university. She has published over 70 journal papers and conference proceedings on various topics related to wireless communications technologies. Her current research interests include radio resource and interference management in mobile communications networks.

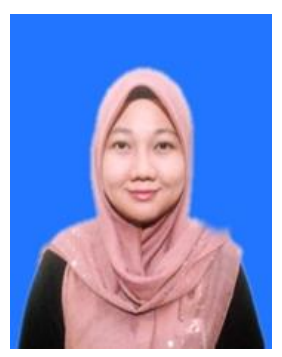

Ainnur Eiza binti Azhar received the Ph.D. degree in Electrical Engineering from the Universiti Teknologi MARA, Malaysia, in 2018. She is currently a Researcher with the Faculty of Electrical Engineering, Universiti Teknologi MARA Shah Alam. Her research interests include PHY channel, interference mitigation technique, LTE-Wi-Fi networks, energy efficiency and green technology. She is a member of Board of Engineer Malaysia and Certified Energy Manager.

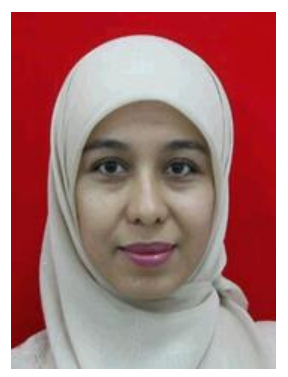

Norsuzila Ya'acob is an Associate Professor in the Department of Communication Engineering, Universiti Teknologi MARA (UiTM). In 2011, she was awarded a Ph.D degree in Electrical, Electronic \& Systems Engineering from University Kebangsaan Malaysia (UKM) for a work on Modeling and Determination of Ionosphere Effects to Improve GPS System Accuracy. She also obtained her M. Sc degree from University Putra Malaysia (UPM) in Remote Sensing and Geographic Information Systems in 2000. Previously, she obtained her B.Eng degree from University Putra Malaysia (UPM), Malaysia in Electronics \& Computer Engineering in 1999. She is a group member of wireless communication technology group (WiCoT) at UiTM and a member of the IEEE Communications Society. She has published over 120 journal papers and conferences proceeding on various topics related to Satellite, Space Weather, Remote Sensing, and Mobile Communication. Her research interests include Satellite, Space Weather, Remote Sensing, Mobile Communication and Signal Processing. 